\title{
A geometric progression on the mass of elementary fermions
}

\author{
L.-G. Mishulan ${ }^{1}$, L. Zhang ${ }^{1} \&$ Q. Xie ${ }^{1}$
}

The Standard Model of particle physics contains three generations of elementary fermions, which have prominent mass hierarchies. Through careful analysis of the observational data on the mass of elementary fermions, we determine that an intrinsic pattern does exist. A geometric progression is proposed to describe the mass distribution of elementary fermions, and using the observational data of neutrino oscillations, we have estimated the neutrino masses for all three generations. At the same time, we propose a hypothesis about the mass distribution of elementary fermions and explore the quantum physical mechanism of the origin of the elementary fermions masses hierarchies.

All three generations of quarks and charged leptons are known to have mass, and previous experiments on neutrino oscillations show that at least two types of neutrinos have mass ${ }^{1,2}$. According to the Standard Model (SM), the masses of elementary particles are observed due to spontaneous symmetry breaking ${ }^{3,4}$. However, the neutrino masses are extremely small. Is the theoretical basis for neutrino masses the same as for the masses of other elementary fermions? In this article, we analyze the observational data of elementary fermion masses and determine the patterns of their mass distribution.

\section{Mass Distribution of Elementary Fermions}

In Table 1, we have arranged the three known generations of quarks and leptons in three columns and four rows in accordance with their masses, and the columns and rows of elementary fermions are denoted by $l$ and $n$, respectively. The first generation of elementary fermions includes a down quark, an up quark, an electron, and an electron neutrino; the second generation--charm quark, strange quark, muon, and a muon neutrino; the third generation--top quark, bottom quark, tau, and tau neutrino (top to bottom); Take the mass of elementary fermions in column $l$ and row $n$ as $m_{l n}$, and put $f_{n}=\left(m_{1 n}+m_{2 n}\right) / m_{3 n}$ and $K_{n}=f_{n} / f_{n+1}$. Using the recommended values from the Particle Data Group $(2020){ }^{5}$, the following values were calculated:

$$
\begin{aligned}
& f_{1}=135.53_{-2.37}^{+2.45} ; f_{2}=44_{-5}^{+3} ; f_{3}=16.7357 \pm 0.0011 \\
& K_{1}=3.085_{-0.242}^{+0.451} ; K_{2}=2.625_{-0.294}^{+0.174}
\end{aligned}
$$

Let $m_{34}=m_{3}, m_{24}=m_{2}, m_{14}=m_{1}, \Delta m_{32}^{2}=m_{3}^{2}-m_{2}^{2}$, and $\Delta m_{21}^{2}=m_{2}^{2}-m_{2}^{1}$, assuming that the neutrino masses are in the normal order. When $m_{1} \ll m_{2}, m_{2} \simeq \sqrt{\Delta m_{21}^{2}}$, then $f_{4} \simeq \sqrt{1+\frac{\Delta m_{32}^{2}}{\Delta m_{21}^{2}}}$.

\footnotetext{
${ }^{1}$ College of Big Data and Information Engineering, Guizhou University, Guiyang 550025, China. Correspondence and requests for materials should be addressed to X.Y.L. (email: miligong@foxmail.com)
} 
Using the data of observed neutrino oscillations ${ }^{5}$, and equations (1), yields:

$$
K_{2} \simeq 2.888 \pm 0.053
$$

We were surprised to find that the values of $K_{1}, K_{2}$, and $K_{3}$ are approximately equal within the error range. Thus, the following hypothesis is proposed: for the mass distribution of the elementary fermions (Table 1 ) $K_{\mathrm{n}}$ is a constant and can be denoted as $K$. Here, it can be called the mass structure constant of elementary fermions. Combining equations (1) and (2), we can concluded that

$$
K=2.866_{-0.196}^{+0.226}, f_{4}=5.839_{-0.427}^{+0.063}
$$

Furthermore, the estimated neutrino masses of three generations are as follows:

$$
\begin{aligned}
& m_{1}=0.0000922_{-0.0000922}^{+0.0006533} \mathrm{eV} ; m_{2}=0.0085737_{-0.0000005}^{+0.0000318} \mathrm{eV} ; \\
& m_{3}=0.0506015_{-0.00000001}^{+0.000054} \mathrm{eV}
\end{aligned}
$$

Table 1 Mass Distribution of Elementary Fermions

$\begin{array}{cccc}\text { Generation } & l=1 & l=2 & l=3 \\ n=1 & d & c & t \\ n=2 & u & s & b \\ n=3 & e & \mu & \tau \\ n=4 & v_{e} & v_{\mu} & v_{\tau}\end{array}$

Geometric progression distribution of elementary fermions masses. In order to better explain the observed data and the pattern of mass distribution of elementary fermions, we propose the following relation:

$$
f_{n}=\left(\frac{1}{\alpha}-1\right) K^{1-n}
$$

where $\alpha$ is the fine structure constant. Using a more accurate mass measurement of charged leptons, the value of $K$ obtained from equation (5) is $2.851050 \pm 0.000096$. This can be viewed as a standard $K$ value. Equation (5) is in very good agreement with observation for $n=1,2$, and 4 .

\section{Discussion and summary}

In this report, we consider equation (5) as a fundamental postulate. Using $\alpha=c \mu_{0} e^{2} / 2 h$, where $e$ is the elementary charge, $\mu_{0}$ is the magnetic constant, $h$ is Planck's constant, and $c$ is the speed of light in a vacuum, equation (5) can be rewritten as follows:

$$
e_{h}^{2}\left(\frac{1}{K^{n-1}}-\frac{1}{K^{n}}\right)=e^{2}\left(\frac{1}{K^{n-1}}-\frac{1}{K^{n}}\right)+e^{2}\left(f_{n}-f_{n+1}\right)
$$

where $\mathrm{e}_{h}= \pm \sqrt{2 h / c \mu_{0}}$, which can be called a vacuum charge. The vacuum charge is considered the basic unit that makes up the vacuum field. We believe that $K^{n}$ in equation (6) characterizes the quantum state of the vacuum field, which can be called a space quantum state. Further, the transition of a vacuum field from one space quantum state to another space quantum state is defined as the space transition of the vacuum field. According to equation (6), when the vacuum field undergoes a space transition, pairs of elementary charges and elementary particles with masses are 
formed. If $f_{\mathrm{n}}=f_{\mathrm{n}+1}$, we obtain $e=e_{h}$. This shows that the presence of elementary fermions with a hierarchy of masses makes the numerical value of elementary charges less than that of vacuum charges. If $f_{\mathrm{n}}$ characterizes some quantum state of an elementary fermion field, then the quantum transition from the state $f_{\mathrm{n}}$ to $f_{\mathrm{n}+1}$ can be defined as a mass transition of the elementary fermion field. From equation (6), we can obtain

$$
e= \pm \sqrt{\frac{2 h}{c \mu_{0}}\left(\frac{1}{1+K^{n} K^{n-1}\left(\frac{f_{n}-f_{n+1}}{K^{n}-K^{n-1}}\right)}\right)}
$$

The above equation shows that elementary charges arise as a result of a space transition in a vacuum field and a mass transition in the elementary fermion field.

\section{References}

1. Abe, S. et al. (KamLAND Collaboration), Precision measurement of neutrino oscillation parameters with KamLAND. Phys. Rev. Lett. 100, 221803 (2008)

2. Ashie, Y.et al. (Super-Kamiokande Collaboration), Measurement of atmospheric neutrino oscillation parameters by Super-Kamiokande I. Phys. Rev. D. 71, 112005 (2005)

3. Nambu, Y. Nobel Lecture: Spontaneous symmetry breaking in particle physics: A case of cross fertilization. Rev. Mod. Phys. 81, 1015 (2009)

4. Peter W. Higgs. Noble Lecture: Evading the Goldstone theorem. Rev. Mod. Phys. 86, 851 (2014)

5. Zyla P. A. et al. (Particle Data Group), Review of particle physics. Prog. Theor. Exp. Phys. 2020, $083 C 01$ (2020).

\section{Acknowledgments}

This work was partially supported by the National Natural Science Foundation of China (Project No. 11963003), the National SKA Program of China (Project No. 2020SKA0110300), the National Key R\&D Program of China (Project No. 2018YFA0404602) and the Youth Science \& Technology Talents Development Project of Guizhou Education Department (Project No. KY[2018]119, [2018]433]).

\section{Author Contributions}

L.-G. Mishulan developed the idea for the study, designed the study, and carried out the study. L. Zhang offered helpful suggestions. Q. Xie supervised the project. All authors discussed the results and commented on the manuscript.

\section{Additional Information}

Competing Interests: The authors declare that they have no competing interests. 